\title{
3-取代苯酞的合成研究进展
}

\author{
李泉城姜岗白瑞韩永康李争宁* \\ (大连大学环境与化工学院 辽宁大连 116622)
}

\begin{abstract}
摘要 含 3-取代苯酞结构的化合物广泛存在于植物和真菌中，是传统中草药中的活性成分，在现代医药中受到广泛关 注. 本综述列举了部分具有生物活性的苯酞类化合物, 综述了 3-取代苯酞类化合物的合成研究进展, 特别是近年来报 道的对映体选择性合成方法，以期对设计、发现具有广泛适用性和高立体选择性地合成苯酞骨架或类似化合物的方法 产生启示作用. 这些方法包括: (a) 通过形成 $\mathrm{C}-\mathrm{C}$ 键的反应构建内酯，例如 2-酰基苯甲酸酯等的醇醛缩合/内酯化级联反 应; (b)通过形成 $\mathrm{C}-\mathrm{O}$ 键的反应构建内酯，例如 2-酰基苯甲酸酯的还原内酯化或 3-烯基苯酞的还原，分子内氧化/内酯 化, 或分子内氧化还原/内酯化. 这些方法对于高立体选择性合成苯唒类化合物和药物研究具有重要意义.

关键词＼cjkstart苯酞；异苯并呋喃-1(3H)-酮; 合成; 对映选择性; 天然产物
\end{abstract}

\section{Progress in the Synthesis of 3-Substituted Phthaides}

\author{
Li, Quancheng Jiang, Lan Bai, Rui Han, Yongkang Li, Zhengning* \\ (College of Environmental and Chemical Engineering, Dalian University, Dalian, Liaoning 116622)
}

\begin{abstract}
Substituted phthalides are widely distributed in plants and fungi. They are active ingredients in traditional Chinese herbal medicines, and have attracted much attention in modern medicinal chemistry. The synthetic methods of 3-substituted phthalides are reviewed, especially those in enantioselective manners. The main approach involves: (a) construction of lactones from $\mathrm{C}-\mathrm{C}$ bond formation reactions, e.g. an aldol/lactonization cascade reaction of 2-acylbenzoates and alikes, (b) construction of lactones via $\mathrm{C}-\mathrm{O}$ bond formation reactions, e.g. reductive lactonization of 2-acylbenzoates or reduction of 3-alkenyl phthalides, intramolecular oxidation/lactonization, or intramolecular redox/lactonization. These methods are of great significance for the high stereoselective synthesis of phthalides and drug research.
\end{abstract}

Keywords phthalide; isobenzofuran-1(3H)-one; synthesis; enantioselectivity; natural product

天然产物在药物研发中发挥了关键作用, 许多现代 药物的开发受到了各种天然产物的启发 ${ }^{[1-2]}$. 苯酞, 即异 苯并呋喃-1(3H)-酮, 为具有 $\gamma$-内酯与苯稠合的双环结构 物质(图 1, 1). 某些植物(如川芎和当归)以及真菌中就含 有这类化合物 ${ }^{[3]}$. 目前, 已分离鉴定出近两百种含苯酞 骨架的天然化合物, 这些化合物在取代基的性质、位置 以及苯环的氧化程度方面表现出差异. 根据苯酞单元的 结构特征, 通常分为三类 ${ }^{[4-5]}$ : (a) 3-未取代的苯酞, (b) 3取代的苯酞和(c)苯酞二聚物. 这些物质显示出重要的 临床特性, 例如抗血小板聚集、抗血栓形成、防止脑缺 血、抗心绞痛、调节心脏功能及中枢神经系统的作 用 ${ }^{[4,6-8]}$. 这类分子的生物活性归因于苯酞中的环内酯结 构 $[9]$.

尽管苯酞类化合物的结构和生物多样性引起了人 们广泛的兴趣, 但是大规模从天然产物中获取这类化合
物并不现实, 因此采用化学方法合成这些化合物具有重 要意义. 本综述将简述苯酞类化合物的结构多样性、生 物活性, 总结在生物活性方面更为重要的 3-取代苯酞的 代表性合成方法, 为相关研究提供参考.

\section{1 生物活性}

具有苯酞结构骨架的霉酚酸(图 1, 2)是一种免疫抑 制剂, 用于器官移植(如肾脏移植)的术后恢复 ${ }^{[10-11]}$; 化 合物 3 能抑制前列腺素 $E_{2}$ 的生成 ${ }^{[12]} ; 4$ 具有抗癌和抗抑 郁活性 ${ }^{[13]}$.

大多数天然苯酞类化合物属于 3 -取代的苯酞(图 1, 5 11), 这些化合物往往具有丰富的药理活性. 具有芹 菜特有气味的丁苯酞 5 (NBP, 图 1), 能够抑制血小板凝 集、改善微循环、减轻缺血性脑损伤, 是治疗缺血性脑 卒中的药物 ${ }^{[14]},(S)$-NBP 在某些情况下效果更佳 ${ }^{[15]}$; 化

* Corresponding author. E-mail: lizhengning@dlu.edu.cn

Received April 30, 2021; revised May 26, 2021; published online July 5, 2021. 
合物 6 具有抗菌活性 ${ }^{[16]} ; 7$ 是 $\mathrm{GABA}_{\mathbf{A}}$ 受体的高效拮抗剂, 用于阻断 $\mathrm{Ca}^{2+}$ 活化的 $\mathrm{K}^{+}$通道 ${ }^{[17]} ; 8$ 具有抗真菌活性, 对 超氧自由基和羟基自由基都起抗氧化作用 ${ }^{[18]} ; \mathbf{9}$ 和 10 是 潜在的植物生长抑制剂[19-20]; 11 对乙酰胆碱酯酶具有抑 制活性 ${ }^{[21]}$.

\section{2 合成路线}

自 1980 年以来, 合成化学家对苯酞类化合物进行 了详细的研究 ${ }^{[22-23]}$. 其合成策略可分为: (a)在苯环上构 建 $\gamma$-内酯, (b)在 $\gamma$-内酯基础上构建碳环, (c)两个环同时 构建. 其中, (a)方案最为重要. 鉴于 3-取代苯酞比 3 位 不含取代基的苯酞结构更为复杂, 合成难度更高, 本综 述重点对 3-取代苯酞类化合物的合成进行总结.

\section{1 形成 $\mathrm{C}-\mathrm{C}$ 键及环内酯的反应}

利用亲核试剂对邻甲酰基苯甲酸或酯的 aldol 加成/ 内酯化反应是合成 3-单取代苯酞最直接的方法之一. 以 含 $\alpha-\mathrm{H}$ 的羰基化合物为亲核试剂, 在酸或碱催化下进行 aldol 反应可形成 $\mathrm{C}-\mathrm{C}$ 键, 接着在酸性介质中进行酯化 反应即可形成 $\gamma$-环内酯(Eq. 1). 2017 年, 贾利娜等 ${ }^{[24]}$ $\beta$-酮酸 $\left(\mathrm{R}^{1}=\mathrm{CO}_{2} \mathrm{H}, \mathrm{R}^{2}=\mathrm{Ar}\right)$ 作为亲核试剂前体, 经茴香 胺脱除其活泼亚甲基质子后与 2-甲酰基苯甲酸反应, 30

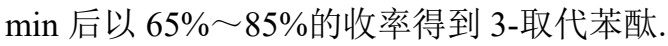

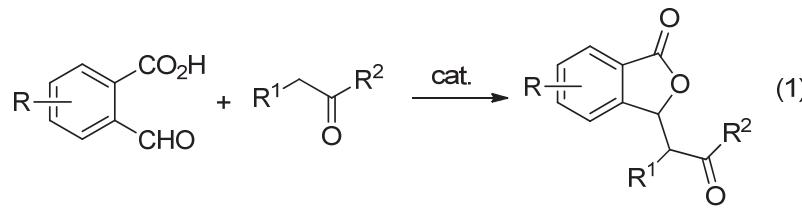

甲磺酸 ${ }^{[25]} 、 \mathrm{ZrOCl}_{2}{ }^{[26]}$ 、蒙脱土 $\mathrm{K}-10^{[27]}$ 、酸性离子 液体 ${ }^{[28]} 、 \mathrm{PhB}(\mathrm{OH})_{2}{ }^{[29]}$ 等均可用于催化反应(Eq. 1). 刘宏 民等 ${ }^{[30]}$ 以苯胺催化反应 $\left(\mathrm{R}=\mathrm{R}^{1}=\mathrm{H}, \mathrm{R}^{2}=\mathrm{Ar}\right)$, 可在较短 的时间生成 3-( $\beta$-羰基)苯酞. 通过 HPLC-MS 分析反应体 系中各组分的 MS 信号, 作者指出反应过程包括苯胺与 醛缩合为席夫碱, 后者进行分子内环合形成 3-胺基苯 酞, 接着在酸性条件下芳基乙酮的烯醇异构体对质子化 的 3-胺基苯酞进行亲核取代反应, 脱去苯胺生成 3-酰甲 基苯酞(Scheme 1).

段文虎等 ${ }^{[31]}$ 以 $2.5 \mathrm{~mol} \%$ 的手性 $L$-脯氨酸酰胺醇 ( $L$-Prolinamide alcohol, 结构式见图 2)催化反应(Eq. 1) $\left(\mathrm{R}=\mathrm{H}, \mathrm{Ph}, \mathrm{OMe}, \mathrm{NO}_{2}, \mathrm{~F}, \mathrm{Cl}, \mathrm{Br} ; \mathrm{R}^{1}=\mathrm{H}, \mathrm{Me} ; \mathrm{R}^{2}=\mathrm{H}, \mathrm{Me}\right.$, $\mathrm{Et})$, 以 $38 \% \sim 91 \%$ 的收率和 $74 \% \sim 97 \%$ 的 $e e$ 值得到 3-( $\beta$-氧代)取代苯酞. 反应需控制在 $-40{ }^{\circ} \mathrm{C}$, aldol 加 成与环化分开进行, 内酯化反应前要先除去前一步反应 的催化剂.

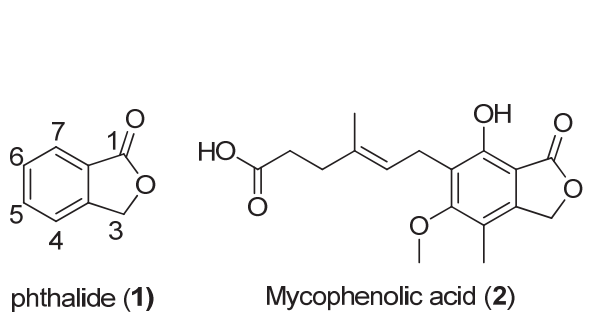

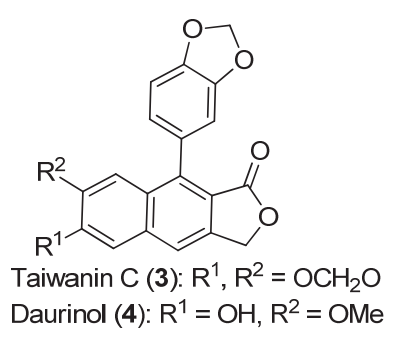

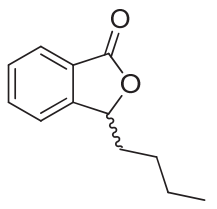

3-butylphthalide (5) ( \pm )-Isoochcacinic acid (6)

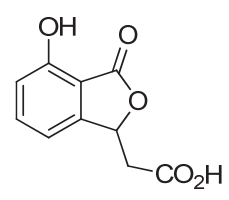
(NBP)<smiles>CCC1OC(=O)c2c1ccc(O)c2OCC(O)CO</smiles>

( \pm )-Chrycolide (10) Phthalide glycerolether (11)<smiles>O=C1OC(c2cccs2)c2cccc(O)c21</smiles><smiles>CN1CCc2cc3c(cc2[C@@H]1[C@H]1OC(=O)c2c1ccc1c2OCO1)OCO3</smiles>

(+)-Bicuculline (7)<smiles>[R]c1cc(O)c(C2OC(=O)c3c(O)cc([R])cc32)c(O)c1</smiles>

Isopestacin (8), $\mathrm{R}^{1}=\mathrm{Me}, \mathrm{R}^{2}=\mathrm{H}$ Cryophonectric acid (9), $\mathrm{R}^{1}=\mathrm{OH}, \mathrm{R}^{2}=\mathrm{CO}_{2} \mathrm{H}$

图 1 苯酞基本结构及部分具有生物活性的苯酞类化合物

Figure 1 Basic structures of phthalide and some biologically active phthalides

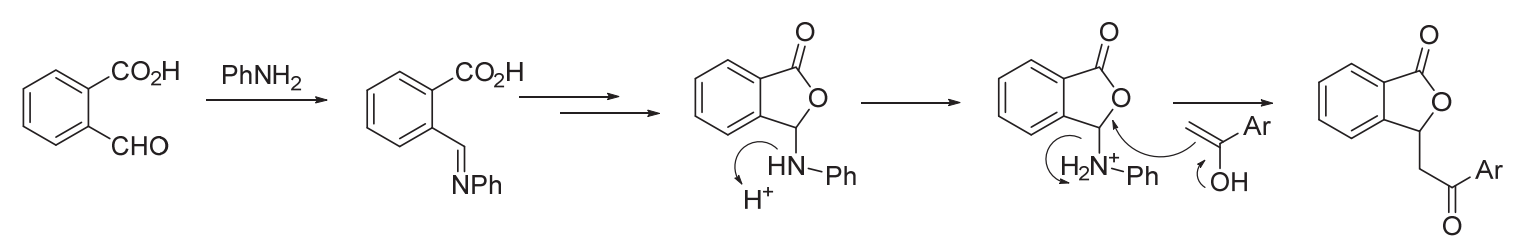

图式 1 苯胺催化 aldol 加成/内酯化反应的可能机理

Scheme 1 Possible mechanism for the aniline-catalyzed aldol/lactonization cascade reaction 
以富电子芳环为亲核试剂, 也可进行类似的反应. 2008 年, 孙兴文等 ${ }^{[32]}$ 利用吲哚与邻甲酰基苯甲酸进行 Friedel-Crafts 反应(Eq. 2), 得到了 3-吲哚取代苯酞; 以 固体酸 Amberlyst ${ }^{[33]}$ 或 $\mathrm{TsOH}^{[34]}$ 作为催化剂也能实现同 样的反应. 2018 年, 赵云辉等 ${ }^{[35]}$ 通过吡啶并咪唑与邻甲 酰基苯甲酸的 Friedel-Crafts 反应合成了 3-咪唑取代苯 酞. 该方法不需要外加催化剂, 可在水中进行, 反应条 件温和, 具有良好的官能团相容性、广泛的底物范围和 较高的原子经济性.

Massa 等[36]用 $\mathrm{K}_{2} \mathrm{CO}_{3}$ 脱除甘氨酸席夫碱的活泼氢并 与邻甲酰苯甲酸甲酯进行 aldol 加成/内酯化串联反应形 成环内酯, 再经过水解, 以良好的产率得到了 3- $(\alpha-$ 氨 基)苯酞.

利用手性催化进行苯酞的对映体合成是近年来高 度活跃的课题. 2019 年, Singh 等 ${ }^{[37]}$ 以 $(R)-\mathrm{H}_{8}-\mathrm{BINOL}$ 磷 酸酯(图 2, Cat.1)催化邻甲酰苯甲酸甲酯与 $\alpha$-重氮酯的 aldol 加成/内酯化串联(Eq. 3), 以良好到优异的产率和 对映选择性生成 3-( $\alpha$-重氮酯)苯酞. 该重氮化合物在 $\mathrm{H}_{2} /$ $\mathrm{PtO}_{2}$ 催化体系中被还原为腙, 产物的 $e e$ 值有所降低; 随 后腙被转化为 $\alpha$-酮酯苯酞，收率为 $70 \%$.

2013 年, Dudding 等[38]采用 $\operatorname{AgF}-(R)-\operatorname{BINAP}$ (图 2, L1)催化邻甲酰基苯甲酸酯的 Sakurai-Hosomi 烯丙基化/ 内酯化(Eq. 4), 以中等收率得到 3-烯丙基苯酞, ee 值
$33 \% \sim 86 \%$, 延长酯基 $\mathrm{R}^{1}$ 的碳链长度可以提高反应的 $e e$ 值. 2018 年, Chen 等 ${ }^{[39]}$ 以烯丙基溴与 Mn 形成的金属有 机试剂为烯丙基化试剂, 在手性 Cr-Pin-BiPy alc (Pineno-bipyridyl alcohol, 结构见图 2)的催化下, 与(取代) 苯甲醛进行与 Eq. 4 类似的 Nozaki-Hiyama-Kishi 烯丙基 化/内酯化反应，以 $70 \% \sim 90 \%$ 的产率和 $94 \% \sim 99 \%$ 的 $e e$ 值生成 3-烯丙基苯酞.

$\mathrm{Hu}$ 等 ${ }^{[40]}$ 采用 $\mathrm{Rh}(\mathrm{I})$ - 手性 1,1'-螺二狮-7,7'-双酚 (SPINOL)的亚磷酸酯(图 2, Cat.2)催化芳基硼酸对邻甲 酰基苯甲酸酯的芳基化(Eq. 5), 以中等到优良的收率和 中等的 $e e$ 值生成 3-芳基苯酞.

2019 年, 宋玲等[41]利用锌(II)-手性 1,2-二苯基乙二 胺单磷酰胺(图 2, L2)的络合物催化芳基硼酸、乙基锌、 邻甲酰基苯甲酸酯的不对称加成/内酯化串联反应，获 得了优异的收率和 $62 \% \sim 87 \%$ 的 $e e$ 值. 反应中芳基硼酸 首先与乙基锌进行转金属化反应，然后进行对邻甲酰基 苯甲酸酯的加成和环合反应. 同年, Ludtke 等 ${ }^{[42]}$ 采用锌手性氨基菜酚配体(图 2, L3) 络合物催化类似的反应，也 得到良好的结果(产率 58\% 95\%, 34\% 91\%ee).

段新方等 ${ }^{[43]}$ 对手性邻碘苯甲酰胺进行卤一镁交换， 借助手性胺进行不对称诱导, 立体选择性地与醛进行加 成形成手性醇，再经分子内环合反应，高对映选择性地 得到 3-单取代苯酞(Scheme 2).

$$
\text { (n), }
$$

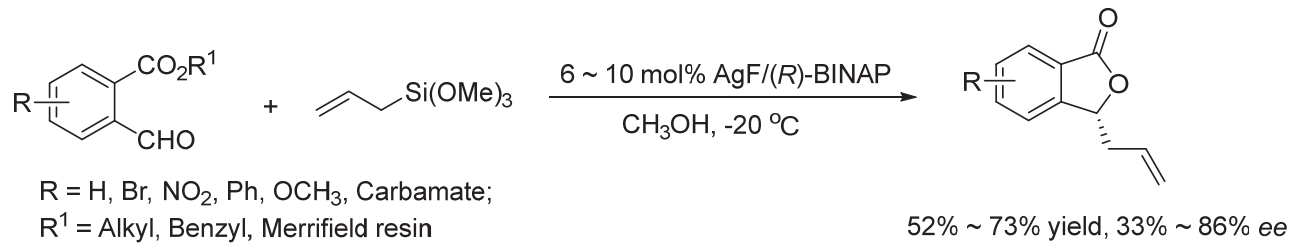


<smiles>O=P(O)(O)Oc1c(Br)cc2c(c1-c1c(Br)cc3c(c1Br)CCCC3)CCCC2</smiles>

$\left(\mathrm{Ar}=2,4,6-\left({ }^{2} \mathrm{Pr}\right)_{3}-\mathrm{C}_{6} \mathrm{H}_{2}\right)$

Cat.1

${ }^{t} \mathrm{Bu}$,<smiles>C[C@H]1COC(c2cnc(C3=N[C@@H](C(C)(C)C)CO3)cn2)=N1</smiles>

PyraBox<smiles></smiles>

$\mathrm{Ar}=2-\mathrm{Naph}$

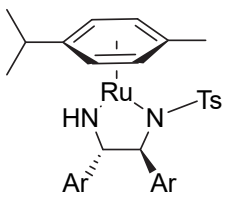

$\mathrm{Ar}=3,5-\left({ }^{\mathrm{t}} \mathrm{Bu}\right)_{2}-\mathrm{C}_{6} \mathrm{H}_{3}$

Cat.3<smiles>CC1(C)C2CCC1C(C(O)(c1ccccc1)c1ccccc1)c1nc(-c3ccccn3)ccc12</smiles>

Pin-BiPy alc

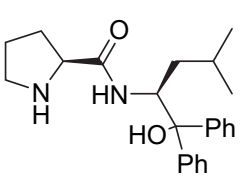

L-Prolinamide alc<smiles>CC(NC([14CH3])c1c(O)ccc2ccccc12)c1ccccc1</smiles>

$\mathrm{Ar}=2-\mathrm{MeC}_{6} \mathrm{H}_{4} \quad$ DPPE

L3

L4<smiles>COc1ccccc1P(CCP(c1ccccc1)c1ccccc1OC)c1ccccc1</smiles>

L1

L2<smiles>CC1(C)c2cccc(-c3ccccc3)c2Oc2c(-c3ccccc3)cccc21</smiles><smiles>c1ccc(-c2ccc3c(c2-c2c(P(c4ccccc4)c4ccccc4)ccc4c2CCCC4)CCCC3)cc1</smiles><smiles>CN1CCOc2c1ccc(-c1ccccc1)c2-c1c(-c2ccccc2)ccc2c1OCCN2C</smiles><smiles>CC1(C)Oc2ccc(-c3ccccc3)c(-c3c(-c4ccccc4)ccc4c3OC(C)(C)O4)c2O1</smiles><smiles>Cc1cccnc1CNc1cccc2c1C1CCc3cccc(c31)O2</smiles>

$(S, S)$-DIPAMP L5

XantPhos

L6

(S)- $\mathrm{H}_{8}$-BINAP L7

(S)-SunPhos

L9
$\mathrm{Ar}=3,5-\left({ }^{t} \mathrm{Bu}\right)_{2} \mathrm{C}_{6} \mathrm{H}_{3}$

$(R)$-SpiroPAP

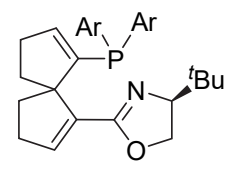

$\mathrm{Ar}=0-\mathrm{Tol}$ $\left(4 S, 5^{\prime} R\right)-$ SpinPHOX L11<smiles></smiles>

$(R, R)-\mathrm{Ph}-\mathrm{BPE}$ L12

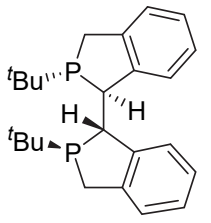

$\left(1 S, 1^{\prime} S, 2 R, 2^{\prime} R\right)$-DuanPhos L13

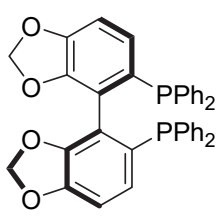

$(R)$-Segphos L14

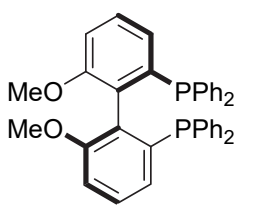

(R)-BIPH-Phos L15

图 2 金属催化合成苯酞所用的的催化剂和配体

Figure 2 Structures of the catalysts and ligands in metal-catalyzed syntheses of phthalides

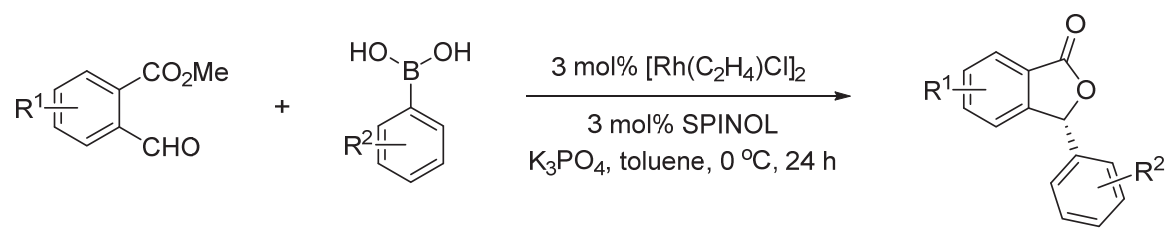

$59 \% \sim 84 \%$ yield, up to $83 \%$ ee

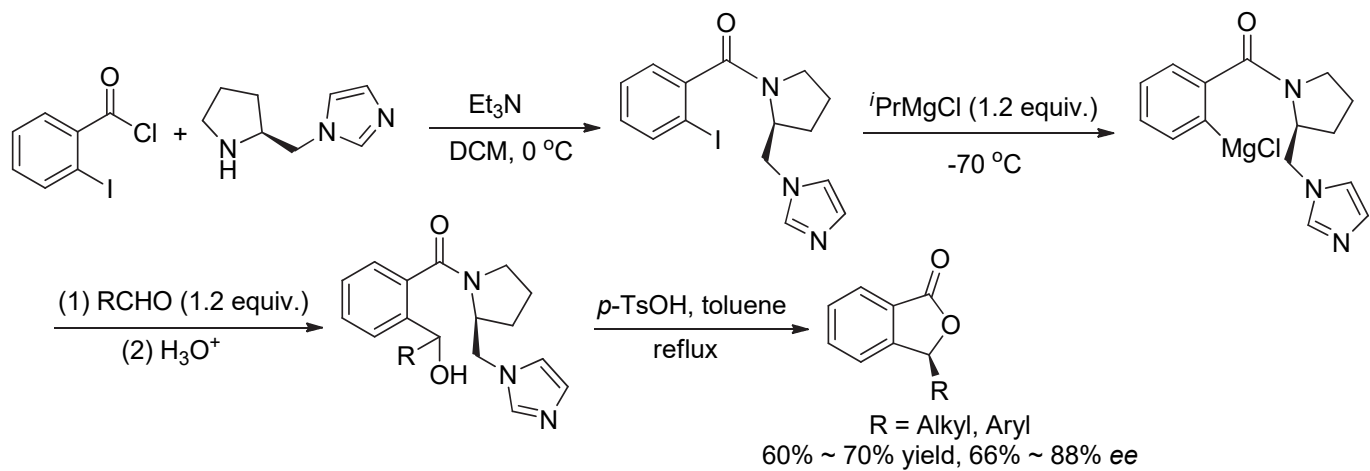

图式 2 亲核取代反应合成手性 3-单取代苯酞

Scheme 2 Synthesis of 3-monosubstituted phthalides via a nucleophilic substitution reaction 
郑建鸿等 ${ }^{[44]}$ 在 $\mathrm{NiBr}_{2}$-DPPE(图 2, L4) 催化下, 将邻 溴苯甲酸甲酯与醛、 $\mathrm{Zn}$ 粉进行反应(Eq. 6), 以中高收率 生成 3-取代苯酞; 而采用邻碘苯甲酸甲酯代替溴代物, 产物收率降低, 这归结于邻碘苯甲酸甲酯脱碘偶联产物 的形成.<smiles>[R]COc1ccc2c(c1)C(=O)OC2[R]</smiles>

采用 $\mathrm{CoI}_{2}-\mathrm{DPPE}$ 为催化剂, 邻碘苯甲酸甲酯与醛、 $\mathrm{Zn}$ 在 THF 中反应, 以优良收率生成相应的苯酞, 这一 方法适于芳香醛和脂肪醛 ${ }^{[45]}$. 以 $(S, S)$-DIPAMP(图 2, L5) 替代 DPPE, 获得最佳的不对称诱导效果, $(S)$-苯酞的收 率为 $81 \% \sim 89 \%$, ee 值为 70\% 98\%. 采用 Pd 催化剂, 邻溴代或碘代苯甲酸与酫、 $\mathrm{Zn}$ 粉反应, 仅形成芳基偶联 产物.

2016 年, Mahendar 和 Satyanarayana 等 ${ }^{[46]}$ 采用 Pd-XantPhos 催化邻溴苄基醇与多聚甲醛反应，合成了 3-取代苯酞(Eq. 7). 其中, 多聚甲酫作为 $\mathrm{CO}$ 源参与反 应，邻溴苄基叔/仲/伯醇均适于该反应. 利用这一方法, 合成了抗血小板聚集药物丁苯酞和细胞毒素受体激动 剂 3-(4-甲氧基)芐基-5,7-二甲氧基苯酞.<smiles>[R]C(O)C1=C(Br)C=C[R1]C=C1</smiles>
$5 \mathrm{~mol} \% \mathrm{Pd}(\mathrm{OAc})_{2}$ $10 \mathrm{~mol} \%$ Xantphos 5 equiv $\left(\mathrm{CH}_{2} \mathrm{O}\right)_{n}$ KOAc, $140^{\circ} \mathrm{C}, 24 \mathrm{~h}$ $\mathrm{R}^{1}=\mathrm{H}$, Alkoxy; $\mathrm{R}^{2}=$ Alkyl

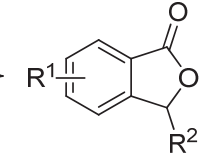

$65 \% \sim 79 \%$ yield
魏俊发等 ${ }^{[47]}$ 通过钉催化芳香酸和芳香醛的分子间

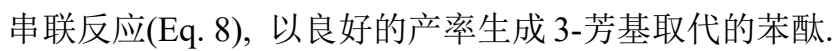
反应过程首先是对芳香酸邻位 $\mathrm{C}-\mathrm{H}$ 键的活化, 其后对 芳香醛的羰基进行加成和分子内酯化. 该方法反应时间
较短、反应物酸和醛易得.

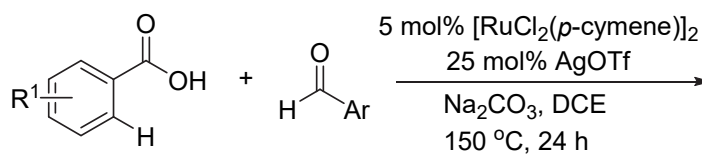

$\mathrm{R}=\mathrm{Me}, \mathrm{OMe}, \mathrm{N}(\mathrm{Me})_{2}, \mathrm{~F}, \mathrm{Cl}$

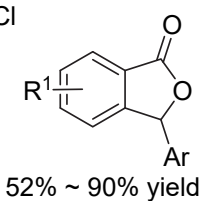

Correia 等 ${ }^{[48]}$ 通过 Pd-Pyrabox 催化芳基重氮盐和 2,3-二氢呋喃的 Heck-Matsuda 芳基化反应，对映体选择 性地合成了 3-取代苯酞(Scheme 3). 反应过程包括 Pd 催 化芳基和烯烃间的偶联反应，然后发生 Pd 迁移反应并 形成稳定的内半缩醛，再经 $\mathrm{NaBH}_{4}$ 还原开环、内酯化反 应得到产物。

2017年, Nguyen 等 ${ }^{[49]}$ 采用 CuI 催化苯并氧杂硅烷的 羧化反应，得到了苯酞(Scheme 4). 反应中无需外加配 体；反应条件温和、操作简便，但底物制备较为困难.

2005 年, Yamamoto 等 ${ }^{[50]}$ 通过 $\mathrm{Cp} * \mathrm{RuCl}$ 催化炔基硼 酸酯、炔丙醇、端基炔的成环三聚反应，生成苯环并硼 氧杂环戌烷，在同样的反应条件下，Pd 催化该中间体与 $\mathrm{CO}$ 反应，生成苯环上带有三个取代基的 3-单取代苯酞 (Scheme 5), 反应具有良好的区域选择性.

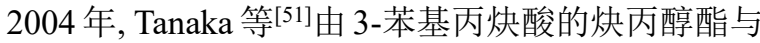
端基炔反应，在 $\mathrm{Rh}-\mathrm{H}_{8}-\mathrm{BINAP}($ 图 2，L7)催化剂作用下， 以较高区域选择性和对映体选择性得到苯酞(Eq. 9), 新 生成的苯环与原有苯环间的旋转受阻, 从而导致产物的

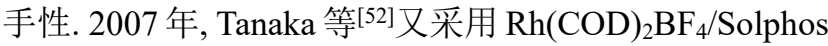
(图 2, L8) 为催化剂, 经 1,6-二炔与炔丙醇的 $[2+2+2]$ 反 应和转移酯化, 对映体选择性地生成手性苯酞, ee 值可 达 94\% (Eq. 10).

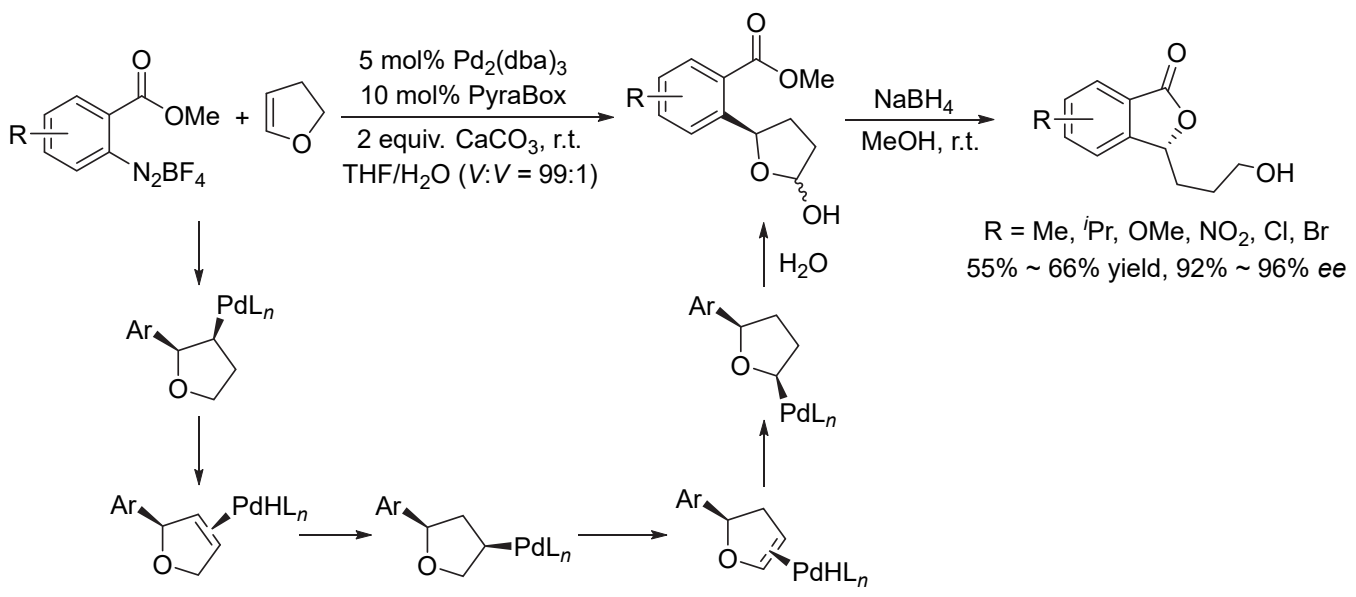

图式 $3 \mathrm{Pd}$ 催化的芳基化/内酯化反应合成手性 3-单取代苯酞

Scheme 3 Synthesis of chiral 3-monosubstituted phthalides via a Pd-catalyzed arylation/lactonization reaction 


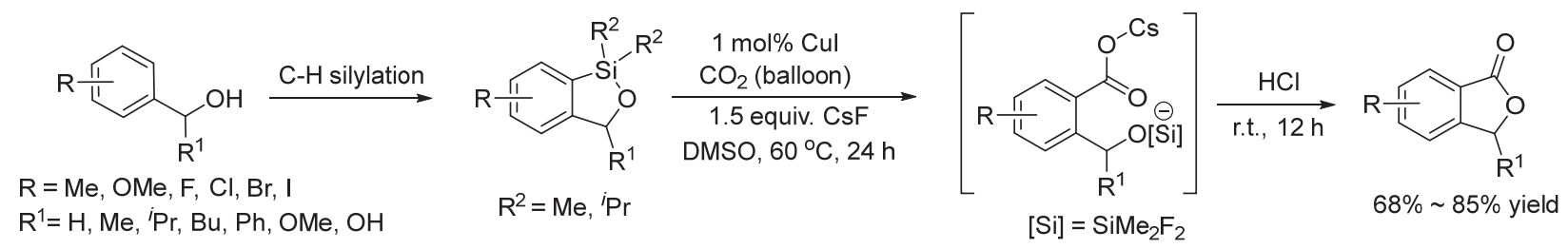

图式 $4 \mathrm{Cu}$ 催化直接羧化合成 3-单取代苯酞

Scheme 4 Synthesis of 3-monosubstituted phthalides via a [Cu]-catalyzed direct carboxylation

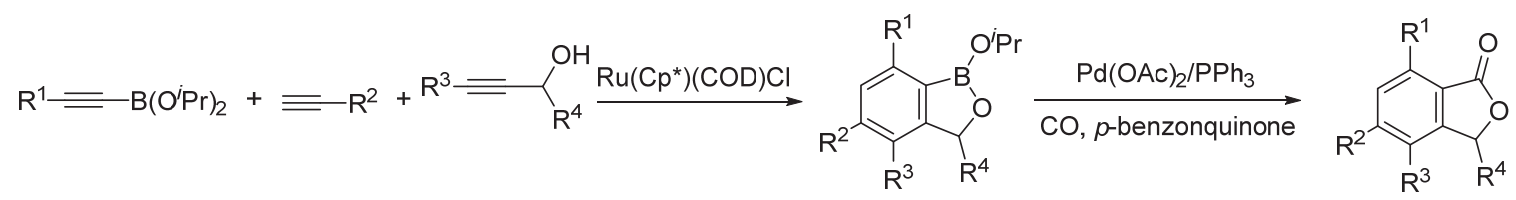

图式 $5 \mathrm{Ru}$ 催化形成苯环的炔烃三聚反应

Scheme 5 Ru-catalyzed formation of benzene framework via cyclotrimerization reaction of alkynes
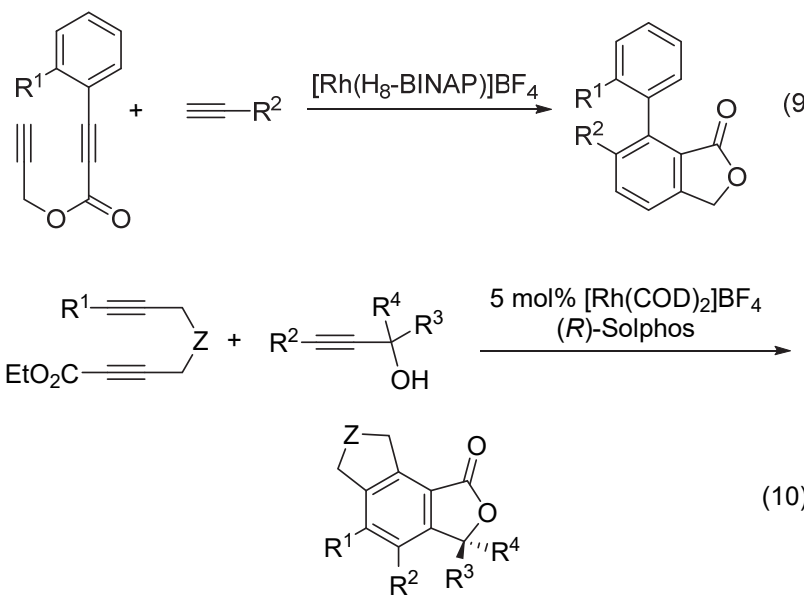

\section{2 形成 $\mathrm{C}-\mathrm{O}$ 键及环内酯的反应}

\subsection{1 还原或还原/内酯化反应}

1988 年, Takaya 和 Noyori 等 ${ }^{[53]}$ 采用 Ru- $(R)$-BINAP 催化邻乙酰基苯甲酸的还原/内酯化反应(Eq. 11)，以 $4.300 \mathrm{MPa}$ 的 $\mathrm{H}_{2}$ 为还原剂, 获得了定量的化学收率和 $92 \%$ 的 $e e$ 值, $(R)$ 构型产物为优势产物. Carpentier 等 ${ }^{[54]}$ 采用 $\left[\mathrm{RuCl}_{2}\left(\eta^{6}\right.\right.$ - $p$-cymene)]-\{TsDPEN $\}$ 催化反应(Eq. 11), 以异丙醇为还原剂和溶剂, 得到 $e e$ 值为 $92 \% \sim 97 \%$ 的 (S)-3-烷基苯酞, 并且产率很高, 但是 3-苯基苯酞的 ee 值仅为 $15 \%$.

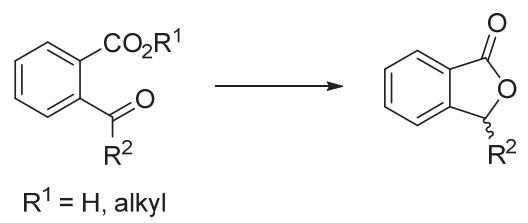

2009 年, 林国强等 ${ }^{[55]}$ 以甲酸钠为还原剂, 采用 1 $\mathrm{mol} \%$ 的 Cat.3(结构见图 2)为催化剂进行 Eq. 11 的反应, 可获得 $90 \% \sim 99 \%$ 的收率和 $98 \% \sim 99 \%$ 的 $e e$ 值. 张兆国
等 ${ }^{[56]}$ 以 Ru- $(S)-S u n P h o s$ (图 2, L9)为催化剂, 以氢气为还 原剂，反应 12４8 h, 得到 58\% 98\%的收率和 34\% $99.6 \%$ 的 $e e$ 值, 催化剂的用量仅为底物的 $0.5 \% .2011$ 年,

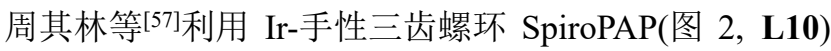
络合物催化邻酰基苯甲酸酯的不对称加氢反应，反应过 程中自发地进行内酯化，以 $98 \%$ 的产率和 $99 \%$ 的 $e e$ 值得 到了 NBP. 值得注意的是, 催化剂用量可降至底物用量 的 $1 / 10^{4}$.

2018 年，丁奎岭等 [58]利用 $\operatorname{Ir}(\mathrm{I})-\operatorname{SpinPHOX}$ (图 2, L11)络合物催化 3-亚烷基苯酞的不对称氢化(Eq. 12), 实现了手性 3-单取代苯酞的合成. 该方法具有广泛的底 物范围; 对于产物 NBP, 可获得 $94 \%$ 的收率和 $95 \%$ 的 ee 值.

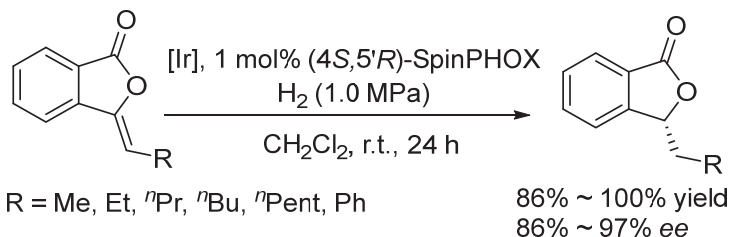

Kawaguchi 等 ${ }^{[59]}$ 报道了碘化氢引发的串联反应. 用 $\mathrm{I}_{2} 、 \mathrm{PPh}_{3} 、 \mathrm{H}_{2} \mathrm{O} 、 2$-炔基苯甲酸甲酯在 $\mathrm{CDCl}_{3}$ 中经一锅反 应(Eq. 13), 以优异的产率生成 3-甲基苯酞. 反应机理如 Scheme 6 所示. 首先, $\mathrm{I}_{2} 、 \mathrm{PPh}_{3}$ 和 $\mathrm{H}_{2} \mathrm{O}$ 生成 $\mathrm{HI}^{[60]}, \mathrm{HI}$ 与 硅基炔烃反应生成末端炔烃; 随后, HI 对末端炔烃加成 生成乙烯基碘化物; 后者迅速消除脱去 MeI 并环化得到 3-亚甲基苯酞; 最后，3-亚甲基苯酞被还原为 3-甲基苯 酞. 该机理为重水实验所证实.

\section{2 .2 分子内氧化/内酯化反应}

Matsuda 等[61]报道了在 $\mathrm{Rh}(\mathrm{III})$ 催化剂和 $\mathrm{Cu}(\mathrm{OAc})_{2}$ 的催化下邻苯二甲醛和醇的氧化/加成/内酯化反应, 生 成 3-烷氧基苯酞(Eq. 14), 产率从中等至良好. 可能的反 
应机理如 Scheme 7 所示, 首先 $[\mathrm{Rh}]$ 对酰基-H 进行插入 反应并脱除 $\mathrm{HCl}$, 形成的酰基氯化铑(III)接着与醇反应 形成甲酰基酯和 $\mathrm{Rh}(\mathrm{I})$; 甲酰基酯与醇形成半缩醛酯, 接着进行分子内环合; 酰基氯化铑(III)中的醛基也可能 与醇反应形成半缩醛, 再对与 $\mathrm{Rh}$ 相连的 $\mathrm{Cl}$ 发生取代反 应形成六元杂环中间体，后者发生还原消除反应生成环 内酯和 $\mathrm{Rh}(\mathrm{I})$. 反应体系中的 $\mathrm{Cu}(\mathrm{II})$ 起氧化 $\mathrm{Rh}(\mathrm{I})$ 为 $\mathrm{Rh}$ (III)的作用. 1,3-二羰基化合物包括羧酸酯可以代替 $\mathrm{ROH}$ 作为亲核试剂，与邻苯二甲醛进行加成/环化反应.

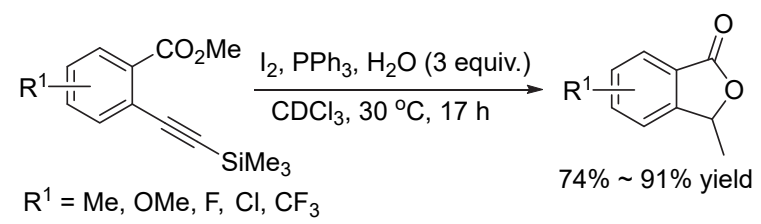

$\mathrm{R}^{1}=\mathrm{Me}, \mathrm{OMe}, \mathrm{F}, \mathrm{Cl}, \mathrm{CF}_{3}$

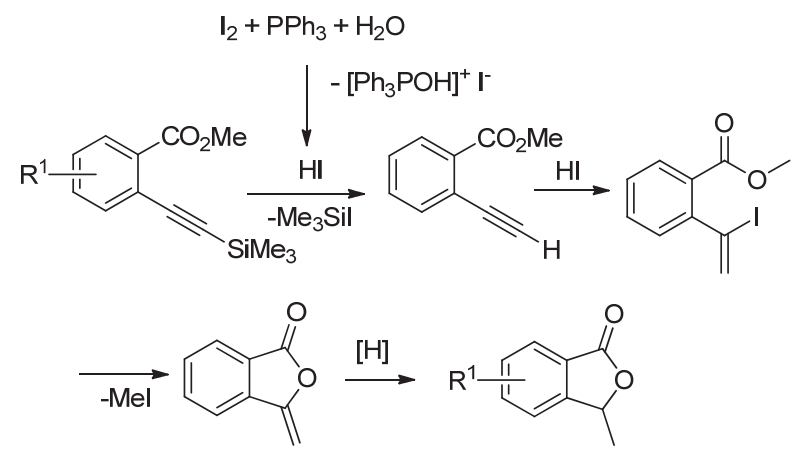

图式 6 碘化氢引发合成 3-单取代苯酞

Scheme 6 Hydroiodination-triggered synthesis of 3-monosubstituted phthalides

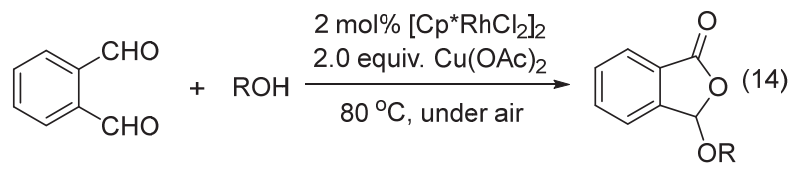

$$
\mathrm{R}=\mathrm{H}, \mathrm{Me}, \mathrm{OMe}, \mathrm{Cl} \quad 40 \% \sim 65 \% \text { yield }
$$
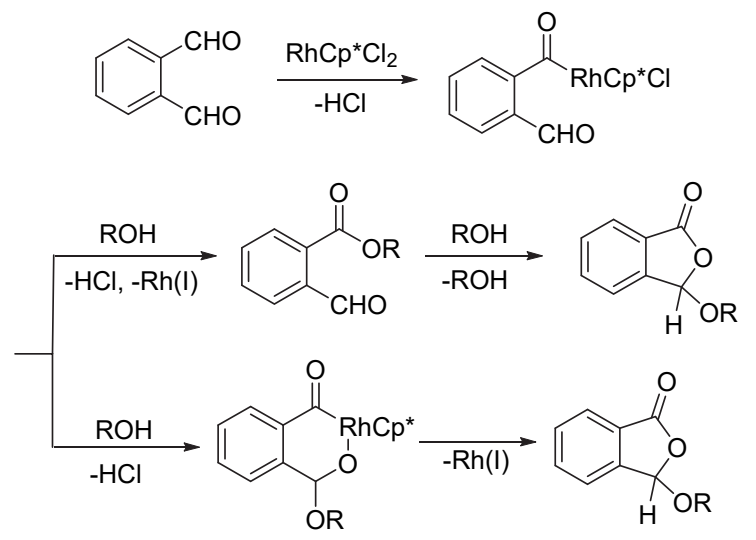

图式 7 Eq. 14 可能的机理

Scheme 7 Proposed mechanism for the Eq. 14
Youn 等 ${ }^{62}$ 报道了三唑盐原位脱除质子形成的 $\mathrm{NHC}$ 催化空气对邻烯基苯甲醛的氧化和 oxa-Michael 加成形 成 3-单取代苯酞的方法(Eq. 15). 该方法具有广泛的底 物范围、对多种官能团具有耐受性, 但催化剂用量较高.

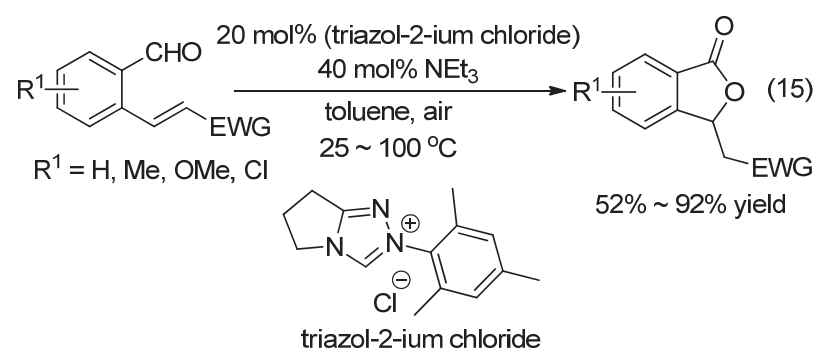

采用手性辛可宁代替 Eq. 15 中的三乙胺, 以 $43 \%$ $90 \%$ 的收率和 $2 \% \sim 86 \%$ 的 $e e$ 值合成 3-单取代苯酞 ${ }^{[63]}$. 辛可宁催化剂作为 Brønsted 碱脱除三唑盐中的质子形 成 $\mathrm{NHC}$, 活化亲核试剂; 其差基作为氢键供体，活化亲 电试剂, 有助于获得优异的对映选择性.

通过氧化邻烷基苯甲酸的苄基位氢也能生成 3-单 取代苯酞. 室温下, $\mathrm{NaBrO}_{3}-\mathrm{NaHSO}_{3}$ 在两相溶剂系统中 与邻烷基苯甲酸反应，以中等到良好的产率一步将邻烷 基苯甲酸转化为 3-单取代苯酞(Scheme 8) ${ }^{[64]}$. 反应过程 可能包括 $\mathrm{HOBr}$ 对苄基氢进行溴代, 羧酸对 $\alpha$-溴代物进 行分子内亲核取代闭环. 含有给电子基团取代的底物进 行反应的收率较低.

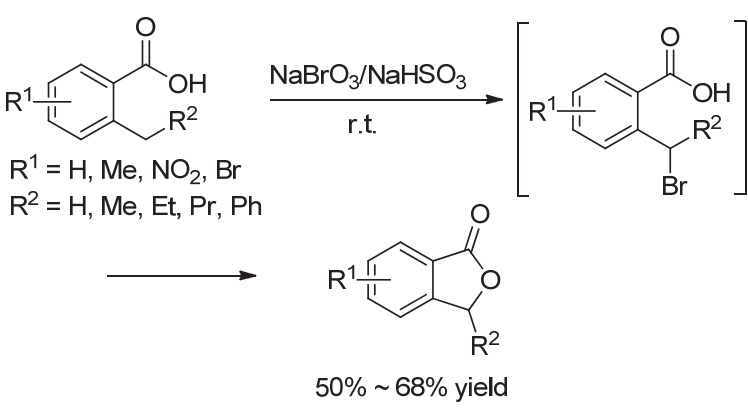

图式 8 氧化/环化反应合成 3-单取代苯酞

Scheme 8 Synthesis of 3-monosubstituted phthalides via an oxidative/cyclization reaction

\section{2 .3 分子内氧化还原/内酯化反应}

芳环相邻位置同时含有醛羰基和酮羰基时，可通过 分子内氧化-还原/内酯化反应生成苯酞(Eq. 16). 2012 年, Schmalz 等 ${ }^{[65]}$ 在 $\mathrm{CN}^{-}$存在下, 对邻甲酰芳基酮进行 Cannizarro-Tishchenko 反应，以中等至良好的产率合成 了苯酞(Scheme 9), 可能的反应机理为: $\mathrm{CN}^{-}$优先进攻醛 基形成氰醇负离子中间体，然后进行分子内氢转移、酮 羰基转化为醇盐中间体，后者对氰基酮进行加成/消除 反应形成内酯环并释放 $\mathrm{CN}^{-}$催化剂。 
<smiles>[R1][R1]1ccc(C(=O)c2cc[R1]([H])cc2C=O)c2c[R1](C)ccc12</smiles><smiles>[R]C(=O)c1ccccc1C=O</smiles>

$\mathrm{R}=\mathrm{Alkyl}$, Aryl $\left(p-\mathrm{Me} / \mathrm{OMe} / \mathrm{NO}_{2} / \mathrm{BrC}_{6} \mathrm{H}_{4}, o-\mathrm{HOC}_{6} \mathrm{H}_{4}, \mathrm{Py}\right)$

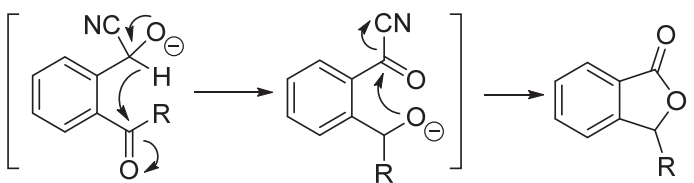

$61 \% \sim 74 \%$ yield

图式 9 Cannizarro-Tishchenko 反应合成 3-单取代苯酞 Scheme 9 Synthesis of 3-monosubstituted phthalides via a Cannizarro-Tishchenko reaction

Yoshikai 等 ${ }^{[6]}$ 以 $\mathrm{CoBr}_{2}$-手性膦配体催化金属对邻酰 基苯酮的 $\mathrm{H}$-酰基化(Eq. 16), 发现 $(R, R)-\mathrm{Ph}-\mathrm{BPE}$ (图 2, L12) 的诱导效果优于 $\left(1 S, 1^{\prime} S, 2 R, 2^{\prime} R\right)$-Duanphos、 $(R, R)-$ Tol-DIPAMP、 $(R, R)$-Me-DuPhos 等配体; 同时, 金属种 类会影响反应的产率和 $e e$ 值, 以 In 为最佳. 采用 10 $\mathrm{mol} \%$ 的 $\mathrm{CoBr}_{2}-(R, R)-\mathrm{Ph}-\mathrm{BPE}$ 和 $20 \mathrm{~mol} \%$ 的 In 反应 $12 \mathrm{~h}$, 以中高收率, $>90 \%$ 的 $e e$ 值得到 3-烷基或苯基取代苯 酞. 将 2- $D$-甲酰基苯乙酮与 2- $H$-甲酰基-5-甲氧基苯乙 酮在同一容器进行反应, D 原子并不会迁移到 5-甲氧基 苯乙酮酮羰基的 $\mathrm{C}$ 原子上, 这表明 $\mathrm{H}$-酰基化反应为分 子内反应而非分子间反应, 即不经过 Tishchenko 机理进 行. 动力学同位素效应 $(1.1 \pm 0.1)$, 表明 $\mathrm{C}-\mathrm{H}$ 的活化并 非速度控制步，因此，还原消除过程为决速步.

Dong 等 ${ }^{[67]}$ 在手性 DuanPhos 双磷配体 (图 2, L13)和 $\mathrm{Ag}^{+}$的存在下，通过 Rh 催化(Eq. 16)获得了 75\% 95\% 的收率和 $92 \% \sim 98 \%$ 的 $e e$ 值. 反应的对映选择性、反应 速率和收率明显受到负离子 $\left(\mathrm{BF}_{4}^{-} 、 \mathrm{NO}_{3}^{-} 、 \mathrm{MsO}^{-}\right.$、 $\mathrm{TfO}^{-}$)影响, 配位能力更高的负离子导致更高的对映选 择性.

Shirai 等 ${ }^{[68]}$ 在 $\operatorname{Ir}-(R)-\operatorname{Seg} \operatorname{Phos}($ 图 2, L14)催化下以 $74 \% \sim 98 \%$ 的 $e e$ 值得到了 3-单取代苯酞产物(Eq. 16). 反 应机理实验表明铱插入醛基 $\mathrm{C}-\mathrm{H}$ 键形成 $\mathrm{Ir}-\mathrm{H}$ 中间体, 再对酮羰基进行加成反应，接着发生还原消除反应生成 环内酯(Scheme 10).

2018 年, Krische 等 ${ }^{[69]}$ 采用 Ir-膦催化邻苯二甲酫的 分子内氧化还原烯丙基化生成 3-烯丙基苯酞(Eq. 17), 可获得 $90 \%$ 以上的 $e e$ 值, 后续可合成 spirolaxine 甲醚和 CJ-12,954 的对映体.

\section{3 天然产物合成}

图 1 列举的天然产物中, 部分已有全合成报道. 本 节总结相关合成路线，其余天然产物的合成路线暂未见 报道.

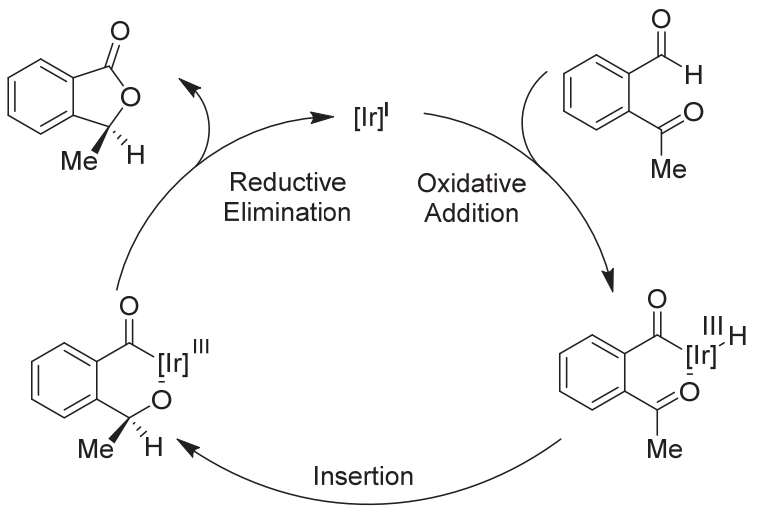

图式 $10 \mathrm{Ir}$ 催化加氢酰化催化循环

Scheme 10 Catalytic cycle of [Ir]-catalyzed hydroacylation

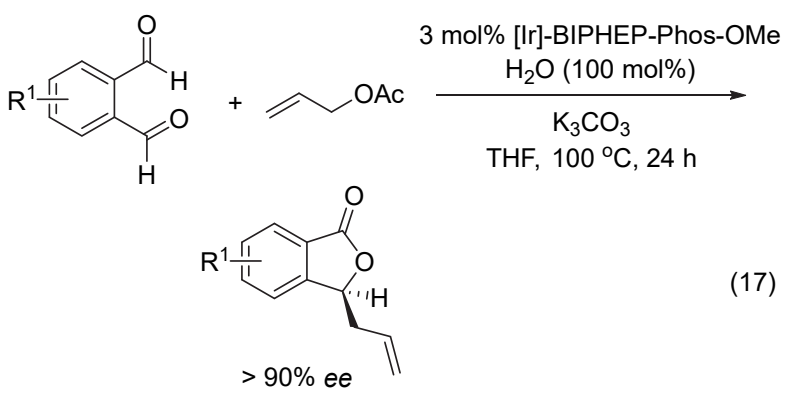

Mycophenolic acid(图 1，2)的合成途径通常是由简 单的底物构建邻苯二甲酸酯，再经过还原/内酯化形成 苯酞骨架，之后通过结构修饰得到产物 [70-71].

Trost 等[72]通过 Horner-Wardsworth-Emmons 反应将 3-羟基-7-甲氧基苯酞转化为 6-羟基-2-(2-羧基乙烯基)苯 甲酸, 然后对苯环上的甲氧基脱甲基并在 1,4-二氮杂二 环[2.2.2]辛烷(DABCO)存在下进行内酯化(Scheme 11), 生成 Isoochracinic acid(图 1,6 ).<smiles>COc1cccc2c1C(=O)OC2O</smiles>

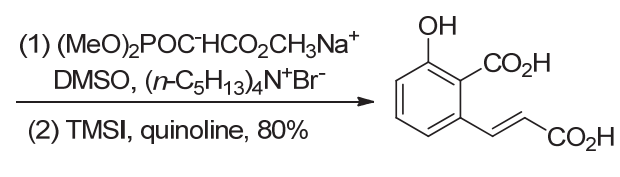<smiles>CC(C)OC(=O)OCC1OC(=O)c2c(O)cccc21</smiles>

图式 11 Isoochracinic acid 的合成

Scheme 11 Synthesis of Isoochracinic acid

如 Scheme 12 所示, Mal 等 ${ }^{[73]}$ 对取代苯甲酰胺依次 
进行甲酰化反应和水解反应, 生成 3-羟基苯酞(邻甲酰 基苯甲酸的半缩醛物), 后者与 1,3-环己二酮发生 Knoevengel 缩合和 Michael 加成, 形成 3-(2,6-二氧代环 己基)苯酞; 再经氧化反应形成 3-芳基苯酞; 最后, 经去 甲基化反应生成 Isopestacin(图 1, 8). 采用类似的方法, 也合成了 Cryophnectric acid(图 1,9).

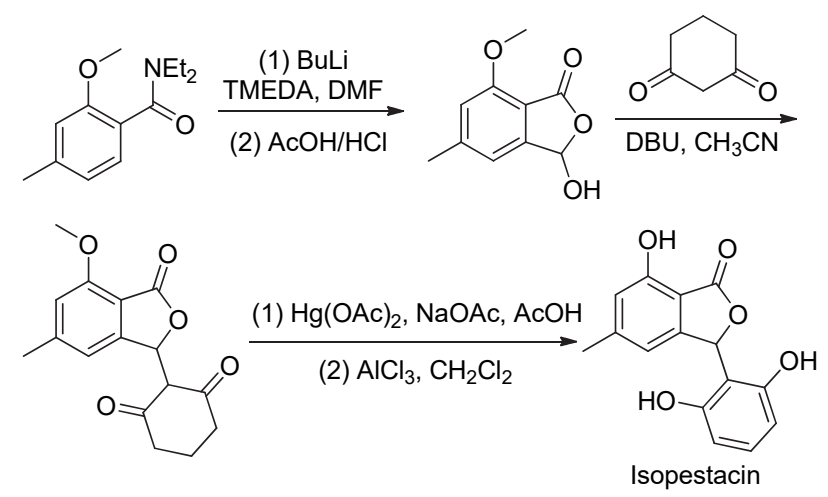

图式 12 Isopestacin 的合成

Scheme 12 Synthesis of Isopestacin

Kuethe 和 Maloney 等 ${ }^{[74]}$ 利用由 2-羟基-6-碘苯甲酸 酯的卤一镁交换形成的取代苯基负离子对醛进行亲核加 成/内酯化串联反应(Eq. 18), 以 $92 \%$ 的产率得到 Chrycolide(图 1, 10). 该方法也适于 $\mathrm{NBP}$ (图 1,5)的合成.

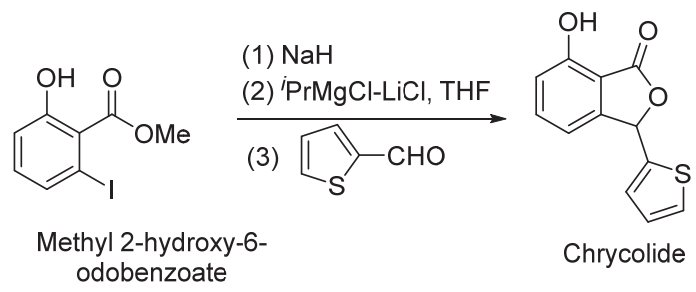

Santos 等[75]对邻溴(取代)苯甲醛进行卤一锂交换和 羧基化反应, 将得到的 3-羟基苯酞用 $\mathrm{NaBH}_{4}$ 还原为苯 酞, 再对苄基位进行脱除质子和硅醚化处理, 生成的硅 醚在 $\mathrm{CsF}$ 作用下与亚胺离子进行反应(Scheme 13), 以 $3.5: 1$ 的质量比得到天然产物 Bicuculline(图 1,7)和其 异构体 Capnoidine.

\section{3 结论}

在过去几十年中，3-取代苯酞的发现和合成受到了 药物化学家和合成化学家的关注. 通过不同的策略合成 了这类化合物, 体现出合成化学的无穷潜力和魅力, 对 合成其他带不同取代基的苯酞具有重要参考价值. 采用 3-亚烷基苯酞的不对称还原或对 2-酰基苯甲酸酯的不对 称还原/内酯化反应合成 3-取代苯酞是最直接的方法, 已经获得了良好的对映体选择性, 在工业生产有重要应
用潜力. 然而，这些优秀结果需在贵金属作用下实现. 因此，发现具有良好选择性特别是立体选择性的廉价金 属催化剂将是今后发展的方向之一.
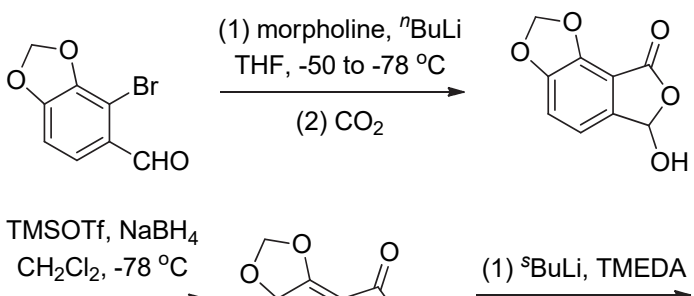<smiles>O=C1OCc2ccc3c(c21)OCO3</smiles>

(1) ${ }^{\text {B BuLi, TMEDA }}$

(2) $\mathrm{TMSCl},-78^{\circ} \mathrm{C}$ THF<smiles>COc1occ2ccc3c(c12)OCO3</smiles>

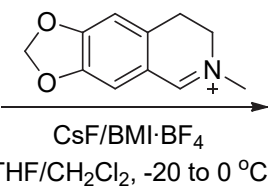<smiles>CN1CCc2cc3c(cc2[C@@H]1[C@H]1OC(=O)c2c1ccc1c2OCO1)OCO3</smiles><smiles>CN1CCc2cc3c(cc2[C@@H]1[C@H]1OC(=O)c2c1ccc1c2OCO1)OCO3</smiles>

图式 13 Bicuculline 的合成 Scheme 13 Synthesis of Bicuculline

\section{References}

[1] Atanasov, A. G.; Zotchev, S. B.; Dirsch, V. M.; Supuran, C. T. Nat Rev. Drug Discovery. 2021, 20, 20.

[2] Cragg, G. M.; Newman, D. J.; Snader, K. M. J. Nat. Prod. 1997, 60, 52.

[3] Hon, P. M.; Lee, C. M.; Choang, T. F.; Chui, K. Y.; Wong, H. N. C. Phytochemistry 1990, 29, 1189.

[4] Lin, G.; Chan, S. K.; Chung, H. S.; Li, S. L. Stud. Nat. Prod. Chem. 2005, 32, 611 .

[5] Zou, J.; Chen, G. D.; Zhao, H.; Huang, Y.; Luo, X.; Xu, W.; He, R. R.; Hu, D.; Yao, X. S.; Gao, H. Org. Lett. 2018, 20, 884.

[6] Beck, J. J.; Chou, S.-C. J. Nat. Prod. 2007, 70, 891.

[7] Brady, S. F.; Wagenaar, M. M.; Singh, M. P.; Janso, J. E.; Clardy, J. Org. Lett. 2000, 2, 4043.

[8] Wang, X.-L.; Wang, Z.-Y.; Yin, J. Zhang, Y.-H. Prog. Pharm. 2016, 40, 89 (in Chinese). (王晓丽, 王兆亚, 尹健, 张奕华, 药学进展, 2016, 40, 89.)

[9] Anderson, W. K.; Boehm, T. L.; Makara, G. M.; Swann, R. T. J. Med. Chem. 1996, 39, 46.

[10] Floryk, D.; Huberman, E. Cancer Lett. 2006, 231, 20.

[11] Chapuis, A. G.; Paolo Rizzardi, G.; D'Agostino, C.; Attinger, A.; Knabenhans, C.; Fleury, S.; Acha-Orbea, H.; Pantaleo, G. Nat. Med. 2000, 6, 762

[12] Ban, H. S.; Lee, S.; Kim, Y. P.; Yamaki, K.; Shin, K. H.; Ohuchi, K. Biochem. Pharmacol. 2002, 64, 1345.

[13] Batsuren, D.; Batirov, É. K.; Malikov, V. M.; Zemlyanskii, V. N.; Yagudaev, M. R. Chem. Nat. Compd. 1981, 17, 223.

[14] Diao, X.; Deng, P.; Xie, C.; Li, X.; Zhong, D.; Zhang, Y.; Chen, X. Drug Metab. Dispos. 2013, 41, 430.

[15] Wang, W.; Cha, X. X.; Reiner, J.; Gao, Y.; Qiao, H. L.; Shen, J. X.; Chang, J. B. Eur. J. Med. Chem. 2010, 45, 1941.

[16] Kameda, K.; Namiki, M. Chem. Lett. 1974, 12, 1491. 
[17] Forgacs, P.; Provost, J.; Touche, A.; Jehanno, A. J. Nat. Prod. 1986, 49, 178.

[18] Strobel, G.; Ford, E.; Worapong, J.; Harper, J. K.; Arif, A. M.; Grant, D. M.; Fung, P. C. W.; Chau, R. M. W. Phytochemistry 2002, $60,179$.

[19] Arnone, A.; Assante, G.; Nasini, G.; Strada, S.; Vercesi, A. J. Nat. Prod. 2002, 65, 48

[20] Tada, M.; Chiba, K. Agric. Biol. Chem. 1984, 48, 1367.

[21] Dai, Y.; Li, K.; She, J.; Zeng, Y.; Wang, H.; Liao, S.; Lin, X.; Yang, B.; Wang, J.; Tao, H.; Dai, H.; Zhou, X.; Liu, Y. Marine Drugs 2020, 18,547

[22] Karmakar, R.; Pahari, P.; Mal, D. Chem. Rev. 2014, 114, 6213.

[23] Li, L. Guangzhou Chem. Ind. 2017, 45, 26 (in Chinese). (李否, 广州化工, 2017, 45, 26.)

[24] Jia, L.; Han, F. Beilstein J. Org. Chem. 2017, 13, 1425.

[25] Limaye, R. A.; Kumbhar, V. B.; Natu, A. D.; Paradkar, M. V.; Honmore, V. S.; Chauhan, R. R.; Gample, S. P.; Sarkar, D. Bioorg. Med. Chem. Lett. 2013, 23, 711.

[26] Maia, A. F. D.; Siqueira, R. P.; de Oliveira, F. M.; Ferreira, J. G.; da Silva, S. F.; Caiuby, C. A. D.; de Oliveira, L. L.; de Paula, S. O.; Souza, R. A. C.; Guilardi, S.; Bressan, G. C.; Teixeira, R. R. Bioorg. Med. Chem. Lett. 2016, 26, 2810.

[27] Landge, S. M.; Berryman, M.; Toeroek, B. Tetrahedron Lett. 2008, 49, 4505 .

[28] Kore, R.; Srivastava, R. Catal. Commun. 2011, 12, 1420.

[29] Palillero-Cisneros, A.; Bedolla-Medrano, M.; Ordonez, M. Tetrahedron 2018, 74, 4174.

[30] Yuan, S.; Wang, S. X.; Chen, J. J.; Zhao, L. F.; Yu, B.; Liu, H. M. Chin. J. Org. Chem. 2018, 38, 309 (in Chinese).

(袁硕, 王四喜, 陈锦杰, 赵龙飞, 余斌, 刘宏民, 有机化学, 2018, 38, 309.)

[31] Zhang, H. Y.; Zhang, S. L.; Liu, L.; Luo, G. S.; Duan, W. H.; Wang, W. J. Org. Chem. 2010, 75, 368.

[32] Lin, H.; Sun, X. W. Tetrahedron Lett. 2008, 49, 5343.

[33] Lin, H.; Han, K. S.; Sun, X. W.; Lin, G. Q. Chin. J. Org. Chem. 2008, 28, 1479 (in Chinese) (林华，韩京成，孙兴文，林国强，有机化学, 2008, 28, 1479.)

[34] Tang, H.; Zhang, X.; Song, A.; Zhang, Z. Mod. Res. Catal. 2012, 1, 11.

[35] Guo, T.; Wang, H. J.; Cao, C. C.; Chen, K. H.; Liu, Y.; Zhang, P. K.; Zhao, Y. H.; Ma, Y. C. Eur. J. Org. Chem. 2020, 12, 3613.

[36] Perillo, M.; Di Mola, A.; Filosa, R.; Palombi, L.; Massa, A. RSC Adv. 2014, 4, 4239.

[37] Ray, S. K.; Sadhu, M. M.; Biswas, R. G.; Unhale, R. A.; Singh, V. K. Org. Lett. 2019, 21, 417.

[38] Mirabdolbaghi, R.; Dudding, T. Tetrahedron 2013, 69, 3287.

[39] Kumbhar, S. V.; Chen, C. RSC Adv. 2018, 8, 41355.

[40] Xing, C. H.; Liao, Y. X.; He, P.; Hu, Q. S. Chem. Commun. 2010, 46, 3010.

[41] Huang, H. Y.; Wang, Y. B.; Zong, H.; Song, L. Appl. Organomet. Chem. 2019, 33, 12

[42] Carlos, A. M. M.; Stieler, R.; Ludtke, D. S. Org. Biomol. Chem. 2019, 17, 283

[43] Zhang, Z. B.; Lu, Y. Q.; Duan, X. F. Synth.-Stuttgart 2011, 3435.

[44] Rayabarapu, D. K.; Chang, H. T.; Cheng, C. H. Chem.-Eur. J. 2004, 10, 2991.

[45] Chang, H. T.; Jeganmohan, M.; Cheng, C. H. Chem.-Eur. J. 2007,
$13,4356$.

[46] Mahendar, L.; Satyanarayana, G. J. Org. Chem. 2016, 81, 7685.

[47] Fan, J.; Wang, P. M.; Wang, J. N.; Zhao, X.; Liu, Z. W.; Wei, J. F.; Shi, X. Y. Sci. China Chem. 2018, 61, 153.

[48] Kattela, S.; de Lucca, E. C.; Correia, C. R. D. Chem.-Eur. J. 2018 24, 17691.

[49] Nguyen, T. V. Q.; Rodriguez-Santamaria, J. A.; Yoo, W. J.; Kobayashi, S. Green Chem. 2017, 19, 2501

[50] Yamamoto, Y.; Ishii, J. I.; Nishiyama, H.; Itoh, K. J. Am. Chem. Soc. 2005, 127, 9625 .

[51] Tanaka, K.; Nishida, G.; Wada, A.; Noguchi, K. Angew. Chem., Int. Ed. 2004, 43, 6510 .

[52] Tanaka, K.; Osaka, T.; Noguchi, K.; Hirano, M. Org. Lett. 2007, 9, 1307.

[53] Kitamura, M.; Ohkuma, T.; Inoue, S.; Sayo, N.; Kumobayashi, H.; Akutagawa, S.; Ohta, T.; Takaya, H.; Noyori, R. J. Am. Chem. Soc. 1988, 110, 629.

[54] Everaere, K.; Scheffler, J. L.; Mortreux, A.; Carpentier, J. F. Tetrahedron Lett. 2001, 42, 1899.

[55] Zhang, B.; Xu, M. H.; Lin, G. Q. Org. Lett. 2009, 11, 4712.

[56] Lu, B.; Zhao, M.; Ding, G.; Xie, X.; Jiang, L.; RatovelomananaVidal, V.; Zhang, Z. ChemCatChem 2017, 9, 3989.

[57] Xie, J. H.; Liu, X. Y.; Xie, J. B.; Wang, L. X.; Zhou, Q. L. Angew. Chem., Int. Ed. 2011, 50, 7329.

[58] Ge, Y.; Han, Z. B.; Wang, Z.; Feng, C. G.; Zhao, Q.; Lin, G. Q.; Ding, K. L. Angew. Chem., Int. Ed. 2018, 57, 13140.

[59] Kawaguchi, S.; Nakamura, K.; Yamaguchi, K.; Sato, Y.; Gonda, Y.; Nishioka, M.; Sonoda, M.; Nomoto, A.; Ogawa, A. Eur. J. Org. Chem. 2017, 2017, 5343.

[60] Kawaguchi, S.; Gonda, Y.; Masuno, H.; Vu, H. T.; Yamaguchi, K.; Shinohara, H.; Sonoda, M.; Ogawa, A. Tetrahedron Lett. 2014, 55, 6779.

[61] Matsuda, T.; Suzuki, K.; Abe, S.; Kirikae, H.; Okada, N. Tetrahedron 2015, 71, 9264.

[62] Youn, S. W.; Song, H. S.; Park, J. H. Org. Biomol. Chem. 2014, 12, 2388.

[63] Youn, S. W.; Song, H. S.; Park, J. H. Org. Lett. 2014, 16, 1028.

[64] Hayat, S.; Rahman, A.; Choudhary, M. I.; Khan, K. M.; Bayer, E. Tetrahedron Lett. 2001, 42, 1647.

[65] Gerbino, D. C.; Augner, D.; Slavov, N.; Schmalz, H. G. Org. Lett. 2012, 14, 2338.

[66] Yang, J.; Yoshikai, N. J. Am. Chem. Soc. 2014, 136, 16748.

[67] Phan, D. H. T.; Kim, B.; Dong, V. M. J. Am. Chem. Soc. 2009, 131, 15608.

[68] Shirai, T.; Iwasaki, T.; Kanemoto, K.; Yamamoto, Y. Chem.-Asian J. 2020, 15, 1858.

[69] Cabrera, J. M.; Tauber, J.; Krische, M. J. Angew. Chem., Int. Ed. 2018, 57, 1390.

[70] Trost, B. M.; Rivers, G. T.; Gold, J. M. J. Org. Chem. 1980, 45 1835.

[71] Patterson, J. W. Tetrahedron 1993, 49, 4789.

[72] Birch, A. J.; Wright, J. J. Aust. J. Chem. 1969, 22, 2635.

[73] Mal, D.; Pahari, P.; De, S. R. Tetrahedron 2007, 63, 11781.

[74] Kuethe, J. T.; Maloney, K. M. Tetrahedron 2013, 69, 5248.

[75] Soriano, M. D. P. C.; Shankaraiah, N.; Santosa, L. S. Tetrahedron Lett. 2010, 51, 1770 . 